\title{
Impact of inner-wall reflection on UV reactor performance as evaluated by using computational fluid dynamics: The role of diffuse reflection
}

\author{
Wentao Li ${ }^{\text {a }}$, Mengkai Li ${ }^{\text {a }}$, James R. Bolton ${ }^{\mathrm{b}}$, Jiuhui Qu ${ }^{\mathrm{a}}$, Zhimin Qiang ${ }^{\mathrm{a},{ }^{3}}$ \\ ${ }^{a}$ Key Laboratory of Drinking Water Science and Technology, Research Center for Eco-Environmental Sciences, University of Chinese Academy of Sciences, \\ Chinese Academy of Sciences, 18 Shuang-qing Road, Beijing, 100085, China \\ ${ }^{\mathrm{b}}$ Department of Civil and Environmental Engineering, University of Alberta, Edmonton, AB T6G 2W2, Canada
}

\section{A R T I C L E I N F O}

\section{Article history:}

Received 28 July 2016

Received in revised form

29 November 2016

Accepted 30 November 2016

Available online 5 December 2016

\section{Keywords:}

UV reactor performance

Inner-wall reflection

Diffuse reflection

Fluence rate distribution

Reduction equivalent fluence

\begin{abstract}
A B S T R A C T
Making use of the reflected ultraviolet (UV) radiation with a reflective inner wall is a promising way to improve UV reactor performance. In this study, the impact of inner-wall reflection on UV reactor performance was evaluated in annular single-lamp UV reactors by using computational fluid dynamics, with an emphasis on the role of diffuse reflection. The UV radiation inside the reactor chamber was simulated using a calibrated discrete ordinates radiation model, which has been proven to be a reliable tool for modeling fluence rate (FR) distributions in UV reactors with a reflective inner wall. The results show that UV reactors with a highly reflective inner wall (Reflectivity $=0.80$ ) had obviously higher FRs and reduction equivalent fluences (REFs) than those with an ordinary inner wall (Reflectivity $=0.26$ ). The inner-wall diffuse reflection further increased the reactor REF, as a result of the elevated volumeaveraged FR. The FR distribution uniformity had conditioned contributions to UV reactor performance. Specifically, in UV reactors with a plug-like flow the FR distribution uniformity contributed to the REF to some extent, while in UV reactors with a mixed flow it had little influence on the REF. This study has evaluated, for the first time, the impact of inner-wall diffuse reflection on UV reactor performance and has renewed the understanding about the contribution of FR distribution uniformity to UV reactor performance.
\end{abstract}

() 2016 Elsevier Ltd. All rights reserved.

\section{Introduction}

Ultraviolet (UV) technologies are being employed worldwide for water disinfection and purification because of their high efficiency in inactivating protozoa and generating oxidative hydroxyl radicals when used in combination with chemicals such as $\mathrm{H}_{2} \mathrm{O}_{2}$ (Hijnen et al., 2006; Esplugas et al., 2002; Xu et al., 2009). Microorganisms or pollutants are inactivated or degraded after receiving a sufficient UV fluence (or UV dose) when flowing through a UV reactor. To ensure an efficient fluence delivery, UV reactors are usually designed with the help of computational fluid dynamics (CFD). Configuration modifications, such as adding baffle plates, have been demonstrated to improve the performance of UV disinfection reactors by providing an optimized reactor

\footnotetext{
* Corresponding author.

E-mail address: qiangz@rcees.ac.cn (Z. Qiang).
}

hydrodynamics (Chiu et al., 1999), and a flow pattern closer to plug flow was found to lead to a higher pollutant degradation in UV reactors for advanced oxidation purpose (Sozzi and Taghipour, 2007). Wols et al. (2011) carried out a comprehensive investigation on the performances of UV reactors with various configurations (i.e., both microbial disinfection and pollutant degradation efficiencies) by taking account of the corresponding fluence distributions. They concluded that measures that increase the average fluence and/or narrow the fluence distribution, such as placing mirrors and adding static mixers, are beneficial to the UV reactor performance.

Among the measures to increase the average fluence of a UV reactor, making use of the reflected UV radiation with a reflector appears to be a promising strategy. Reflection can be of two types: specular reflection, where the UV light is reflected like that at a mirror; and diffuse reflection, where the UV light is reflected from a ragged surface in all directions. Sommer et al. (1996) found that the use of an aluminum reflector increased the reduction equivalent 


\begin{tabular}{|c|c|c|c|}
\hline \multicolumn{2}{|c|}{ Nomenclature } & $\mathrm{FR}_{\min }$ & Minimum fluence rate $\left(\mathrm{mW} \mathrm{cm}^{-2}\right)$ \\
\hline$A$ & Surface area of quartz sleeve $\left(\mathrm{m}^{2}\right)$ & $\mathrm{FR}_{\mathrm{wa}}$ & Weighted average fluence rate $\left(\mathrm{mW} \mathrm{cm}^{-2}\right)$ \\
\hline CFD & Computational fluid dynamics & HRW & Highly reflective inner wall \\
\hline $\mathrm{CF}_{\mathrm{i}}$ & Individual calibration factor $(-)$ & $k$ & Microbial UV inactivation rate constant $\left(\mathrm{cm}^{2} \mathrm{~mJ}^{-1}\right)$ \\
\hline $\mathrm{CF}_{\mathrm{u}}$ & Uniform calibration factor $(-)$ & $L_{1}$ & UV reactor length $(\mathrm{mm})$ \\
\hline$d_{1}$ & Reactor internal diameter (mm) & $L_{2}$ & Quartz sleeve length (mm) \\
\hline$d_{2}$ & Reactor inlet (or outlet) internal diameter (mm) & $L_{3}$ & Lamp arc length $(\mathrm{mm})$ \\
\hline DO & Discrete ordinates & MFSD & Micro-fluorescent silica detector \\
\hline e & Internal emissivity - emission of thermal radiation $(-)$ & $N$ & Total simulating particle number $(-)$ \\
\hline$E_{\mathrm{o}}$ & Direct Irradiation on the radiation boundary $\left(\mathrm{W} \mathrm{m}^{-2}\right)$ & ORW & Ordinary inner wall \\
\hline$f_{\mathrm{d}}$ & Diffuse fraction of inner-wall reflection $(-)$ & $P$ & Lamp power $(\mathrm{W})$ \\
\hline$F_{0}$ & $\begin{array}{l}\text { Intercept of microbial fluence-response curve on } \mathrm{X} \text { axis } \\
\left(\mathrm{mJ} \mathrm{cm} \mathrm{cm}^{-2}\right)\end{array}$ & $\begin{array}{l}\text { PDR } \\
R\end{array}$ & $\begin{array}{l}\text { Partially diffuse reflection } \\
\text { Reflectivity }(-)\end{array}$ \\
\hline$F_{\mathrm{i}}$ & Fluence received by the $i^{\text {th }}$ particle $\left(\mathrm{mJ} \mathrm{cm}^{-2}\right)$ & $R_{\mathrm{d}}$ & Diffuse reflectivity $(-)$ \\
\hline$F_{\min }$ & Particle minimum fluence $\left(\mathrm{mJ} \mathrm{cm}^{-2}\right)$ & REF & Reduction equivalent fluence $\left(\mathrm{mJ} \mathrm{cm} \mathrm{cm}^{-2}\right)$ \\
\hline FDR & Fully diffuse reflection & RSD & Relative standard deviation $(-)$ \\
\hline FR & Fluence rate $\left(\mathrm{mW} \mathrm{cm}^{-2}\right)$ & UVT & UV transmittance $(\%)$ \\
\hline $\mathrm{FR}_{\mathrm{aa}}$ & Area-averaged fluence rate $\left(\mathrm{mW} \mathrm{cm}^{-2}\right)$ & $\eta$ & Lamp UVC efficiency (\%) \\
\hline $\mathrm{FR}_{\max }$ & Maximum fluence rate $\left(\mathrm{mW} \mathrm{cm}^{-2}\right)$ & & \\
\hline
\end{tabular}

fluence (REF) of a UV disinfection device by over $40 \%$ at a water UV transmittance (UVT) of $97 \%$. Recently, with a rapid progress in UV light emitting diodes (UV-LEDs), UV reactors employing this new light source have been developed, and reflectors made of aluminum or other highly reflective materials have been applied to make the best use of UV radiation inside the reactor chamber (Hessling et al., 2016; Wurtele et al., 2011). Nevertheless, researches concerning how the reflected UV radiation contributes to the UV reactor performance are still very limited, compared to the abundant works discussing the effect of the reactor hydrodynamics (Sozzi and Taghipour, 2006a; Wols et al., 2011; Xu et al., 2013).

Two obstacles lie in the way of an in-depth understanding of the effect of the reflected UV radiation. On the one hand, many commonly used fluence rate (FR) distribution models, such as the multiple segment source summation model whose reliability has been widely accepted, do not include a component to calculate the reflected UV radiation; on the other hand, although some FR distribution models, such as the discrete ordinates (DO) radiation model, do account for the reflection from the reactor inner wall, their accuracies in FR distribution predictions have not been well evaluated (Liu et al., 2004). In fact, there have been contradictory results with regard to the accuracy of the DO radiation model. Liu et al. (2004) found that the DO radiation model significantly overestimated the FRs in the near-lamp region but underestimated the FRs in the near-wall region; however, Ho (2009) found that the predicted FR distributions by the DO radiation model agreed quite well with the experimental data.

Recently, Li et al. (2011) developed a micro-fluorescent silica detector (MFSD) with a small volume and $360^{\circ}$ response, which is very suitable for in-situ measurements of FR distributions inside UV reactors. Consequently, the FR distributions predicted by numerical models can be readily verified with the MFSD measurements, even in UV reactors with a reflective inner wall (Li et al., 2012). Based on the in-situ measured FR data, they found that the weighted average $\mathrm{FR}\left(\mathrm{FR}_{\mathrm{wa}}\right)$ was almost doubled in a UV reactor when aluminum was used as the inner-wall material in place of stainless steel. Another important finding is that the diffuse reflection of the inner wall enhanced the FR distribution uniformity, and a more uniform FR distribution was believed to improve the UV reactor performance (Li et al., 2012). From this perspective, the inner wall of UV reactors should be made of materials with a high diffuse reflectivity. Nevertheless, to date no further study has been conducted to elucidate the effects of inner-wall diffuse reflection on FR distributions and the resulting UV reactor performance.

In this study, the impact of inner-wall reflection on UV reactor performance was evaluated in annular single-lamp UV reactors by using CFD simulations, with an emphasis on the role of diffuse reflection. FR distributions inside the reactor chamber were predicted by a DO radiation model after being calibrated with in-situ measured FR data. The effects of inner-wall reflection on FR distributions and REF were examined with different inner walls, and the underlying reason for a better performance of UV reactors with a higher inner-wall diffuse reflection was explored. This study, for the first time, has evaluated the impact of inner-wall diffuse reflection on UV reactor performance and clarified the contribution of FR distribution uniformity.

\section{Materials and methods}

\subsection{UV reactors}

Two annular single-lamp UV reactors with different inlet and outlet positions were employed as the model UV reactors, which were denoted as the L-shape (Fig. 1a) and U-shape (Fig. 1b) UV reactors, respectively. The different reactor configurations lead to different hydrodynamics inside the reactors. The UV reactors had a length $\left(L_{1}\right)$ of $400 \mathrm{~mm}$ and an internal diameter $\left(d_{1}\right)$ of $95 \mathrm{~mm}$. The length $\left(L_{2}\right)$ of the quartz sleeve ( $23 \mathrm{~mm}$ o.d., $>98 \%$ transmittance at $254 \mathrm{~nm}$ ) was $347 \mathrm{~mm}$ while the lamp arc length $\left(L_{3}\right)$ was $297 \mathrm{~mm}$. The low-pressure mercury lamp (GL Type, XiashiWanhua Co., China) had an output power $(P)$ of $16 \mathrm{~W}$ and UVC efficiency $(\eta$, at $254 \mathrm{~nm}$ ) of $26 \%$. The inlet and outlet had a same internal diameter $\left(d_{2}\right)$ of $45 \mathrm{~mm}$, and the distance from the outlet central axis to the reactor end was $50 \mathrm{~mm}$. Two kinds of inner walls were simulated, that is, an ordinary inner wall (ORW) with a reflectivity $(R)$ of 0.26 (typical of stainless steel) and a highly reflective inner wall (HRW) with $R=0.80$ (typical of aluminum). The diffuse fraction $\left(f_{\mathrm{d}}\right)$ of the inner-wall reflection varied from 0.1 to 0.9 , accounting for inner walls with various diffuse reflectivities. 


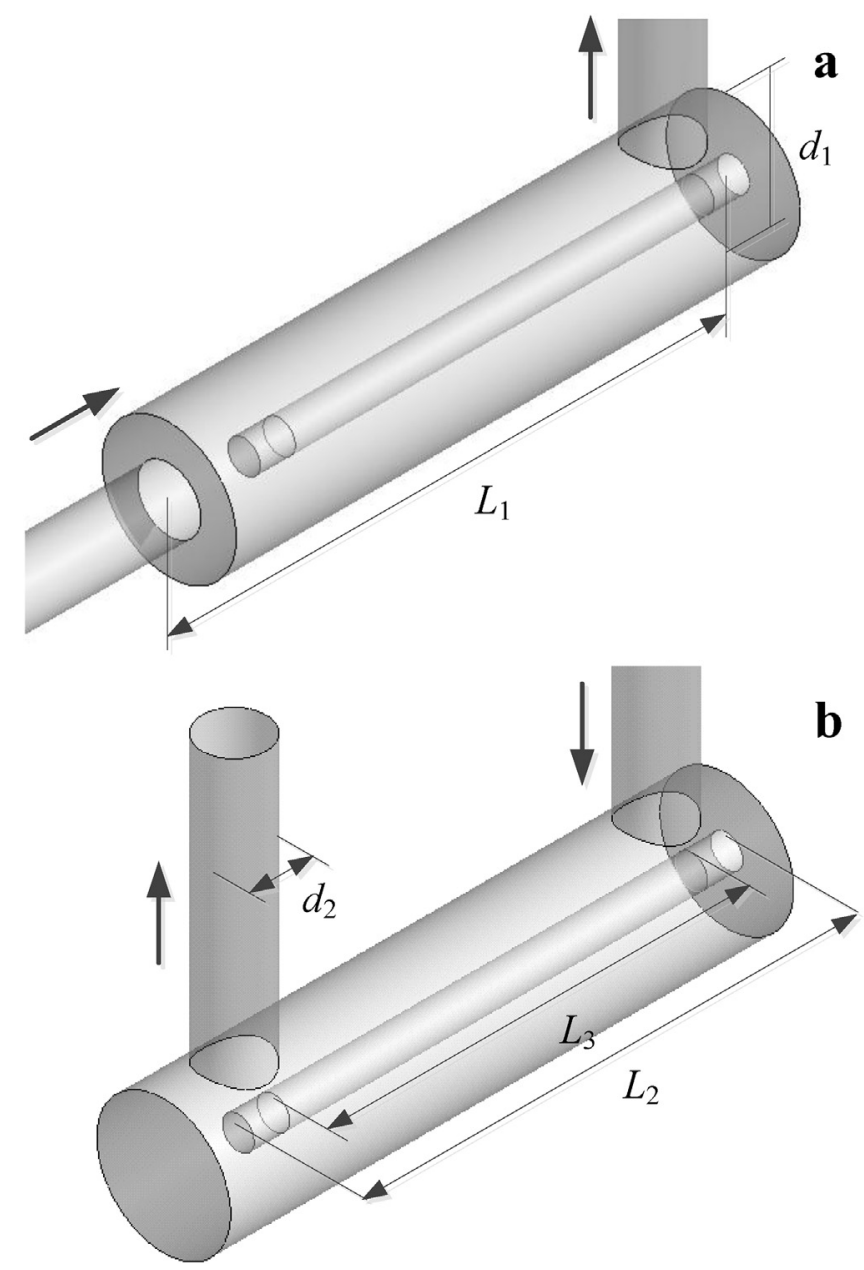

Fig. 1. Schematic diagram of the modeled UV reactors: (a) L-shape; and (b) U-shape.

\subsection{Model settings}

The hydrodynamics inside the UV reactors were modeled with the Realizable $k-\varepsilon$ model, which has been proven to produce a reliable simulation of the flow field in annular UV reactors (Sozzi and Taghipour, 2006b). Unless otherwise specifically stated, the velocity at the reactor inlet was set at $0.1 \mathrm{~m} \mathrm{~s}^{-1}$, which corresponded to a flow rate of $0.57 \mathrm{~m}^{3} \mathrm{~h}^{-1}$. The water UV transmittances were set at $99 \%, 90 \%$ and $75 \%$, corresponding to the absorption coefficients of $1.0,10.5$ and $28.8 \mathrm{~m}^{-1}$, respectively, in the model boundary conditions. The UV radiation was simulated by the DO radiation model, with the UV light assumed to be emitted from the quartz sleeve (Ho, 2009). According to the Fluent Manual (ANSYS, 2010), the reflectivity of an inner wall can be determined as follows:

$R=1-e$

$R_{d}=f_{d}(1-e)$

where $e=$ internal emissivity of the inner wall (i.e., emission of thermal radiation), $R_{\mathrm{d}}=$ diffuse reflectivity. Consequently, the various inner-wall reflections can be simulated by setting the $e$ and $f_{\mathrm{d}}$ values accordingly. The $e$ values were set at 0.74 and 0.20 for the ORW and HRW, respectively. Low, moderate and high inner-wall diffuse reflections were obtained by setting the $f_{\mathrm{d}}$ values at 0.1 , 0.5 and 0.9 , respectively. The microbial transportation and inactivation were simulated with the Lagrangian approach of the
Discrete Phase model by releasing more than 5000 simulating particles at the reactor inlet, and then their received fluences were calculated by a complied user-defined function (Ho et al., 2009). The REFs of the UV reactors were calculated following Eq. (3), assuming that the fluence-response of the challenge microorganism follows the delayed Chick-Watson model:

$\mathrm{REF}=-\frac{1}{k} \lg \left(\frac{1}{N} \sum_{i=1}^{N} 10^{-k\left(F_{i}-F_{0}\right)}\right)+F_{0}$

where $k=$ inactivation rate constant of the challenge microorganism $\left(\mathrm{cm}^{2} \mathrm{~mJ}^{-1}\right), F_{0}=$ intercept of the fluence-response curve on the $\mathrm{X}$ axis $\left(\mathrm{mJ} \mathrm{cm} \mathrm{cm}^{-2}\right), N=$ number of the total simulating micro particles, $F_{\mathrm{i}}=$ fluence received by the $i^{\text {th }}$ particle $\left(\mathrm{mJ} \mathrm{cm}{ }^{-2}\right)$. The challenge microorganism employed in this study was Bacillus subtilis spores (ATCC 6633). According to our previous study, the values of $k$ and $F_{0}$ were determined to be $0.087 \mathrm{~cm}^{2} \mathrm{~mJ}^{-1}$ and $3.0 \mathrm{~mJ} \mathrm{~cm}^{-2}$, respectively (Qiang et al., 2013).

All the modeling processes were accomplished with the commercial CFD software package of ANSYS Fluent 13.0 (Canonsburg, PA, USA), and convergence of the solution was reached if the normalized residuals were all at a level of $10^{-5}$ or less. The model settings are detailed in Text S1 (see Supplementary Information).

\subsection{Model calibration}

Since there have been disputes about the accuracy of the DO radiation model in predicting FR distributions in UV reactors, a calibration was first conducted using the in-situ measured FR data. Instead of comparing the FRs of individual points inside the UV reactors as practiced by Qiang et al. (2013), $\mathrm{FR}_{\mathrm{wa}}$ was introduced in this study to calculate the calibration factor. This ensured an overall consistence of the results from the calibrated radiation model with the experimental data. The FRs of 11 test points with a radial distance to the sleeve ranging from 3 to $28 \mathrm{~mm}$ were employed to calculate the $\mathrm{FR}_{\mathrm{wa}}$ by considering their respective weighting factors (Text S2, see Supplementary Information) (Li et al., 2012). These test points were equidistantly distributed in the reactor chamber (Fig. S1) and thus could well represent the cross-sectional FR variations. The calibration factor for the DO radiation model was then determined as the ratio of the $\mathrm{FR}_{\mathrm{wa}}$ measured by the MFSD to that predicted by the model. Since the in-situ FR measurements were conducted in UV reactors with inner walls of a small diffuse reflectivity (Li et al., 2012), the $f_{\mathrm{d}}$ was set at 0.1 in the DO radiation model during the calibration process.

\section{Results and discussion}

\subsection{Calibration of DO radiation model}

The DO radiation model exhibited a general overestimation of the FRs in the UV reactors (Fig. S2), thus the calibration was very necessary. Table 1 presents the $\mathrm{FR}_{\mathrm{wa}}$ values determined by the MFSD (FR $\left.{ }_{\mathrm{wa}}-\mathrm{MFSD}\right)$ and the DO radiation model $\left(\mathrm{FR}_{\mathrm{wa}}-\mathrm{DO}\right)$ for UV reactors with two inner-wall reflectivities $(R=0.26$ and 0.80$)$ at various water UVTs as well as the corresponding calibration factors. The individual calibration factors $\left(\mathrm{CF}_{\mathrm{i}}\right)$ ranged from 0.77 to 0.84 at high UVTs (i.e., $90 \%$ and $99 \%$ ) and 0.91 to 0.92 at UVT of $75 \%$. Because the effect of inner-wall reflection was notable only at high UVTs, a uniform calibration factor $\left(\mathrm{CF}_{\mathrm{u}}\right)$ of 0.80 was adopted, mainly accounting for the $\mathrm{CF}_{\mathrm{i}}$ values at UVTs of $90 \%$ and above (typical for drinking water). The $\mathrm{CF}_{\mathrm{u}}$ was then introduced into the Direct Irradiation calculations in the boundary condition settings (Eq. (4)), which caused a $20 \%$ reduction in the overall FR as calculated by the 
Table 1

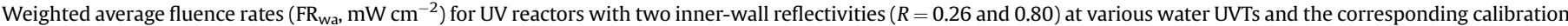
factors for the DO radiation model. The diffuse fraction $\left(f_{\mathrm{d}}\right)$ was set at 0.1 in the DO radiation model.

\begin{tabular}{|c|c|c|c|c|c|c|}
\hline \multirow[t]{2}{*}{ Parameter } & \multicolumn{3}{|c|}{$R=0.26$} & \multicolumn{3}{|c|}{$R=0.80$} \\
\hline & $99 \%$ & $90 \%$ & $75 \%$ & $99 \%$ & $90 \%$ & $75 \%$ \\
\hline $\mathrm{FR}_{\mathrm{wa}}-\mathrm{MFSD}$ & 10.76 & 7.35 & 4.65 & 23.18 & 10.20 & 5.42 \\
\hline $\mathrm{FR}_{\mathrm{wa}}-\mathrm{DO}$ & 13.35 & 8.78 & 5.06 & 30.25 & 13.20 & 5.93 \\
\hline $\mathrm{CF}_{\mathrm{i}}$ & 0.81 & 0.84 & 0.92 & 0.77 & 0.77 & 0.91 \\
\hline $\mathrm{CF}_{\mathrm{u}}$ & & & & & & \\
\hline
\end{tabular}

$\mathrm{FR}_{\mathrm{wa}}-\mathrm{MFSD}$ : $\mathrm{FR}_{\mathrm{wa}}$ determined by the MFSD; $\mathrm{FR}_{\mathrm{wa}}-\mathrm{DO}$ : $\mathrm{FR}_{\mathrm{wa}}$ determined by the $\mathrm{DO}$ radiation model; $\mathrm{CF}_{\mathrm{i}}$ : individual calibration factor; and $\mathrm{CF}_{\mathrm{u}}$ : uniform calibration factor.

calibrated DO radiation model.

$E_{o}=\mathrm{CF}_{\mathrm{u}} \frac{P \eta}{A}$

where $E_{0}=$ Direct Irradiation on the radiation boundary $\left(\mathrm{W} \mathrm{m}^{-2}\right)$, $P=$ lamp power (W), $\eta=$ lamp UVC efficiency, $A=$ surface area of the quartz sleeve $\left(\mathrm{m}^{2}\right)$.

The radial FR distributions in the central planes of UV reactors with two inner-wall reflectivities at various water UVTs are shown in Fig. 2. The predicted FR distributions by the calibrated DO radiation model agreed well with the measured data by the MFSD, regardless of the inner-wall reflectivities. At UVTs of $90 \%$ and $99 \%$, the relative deviations between the predicted and measured $\mathrm{FR}_{\mathrm{wa}}$ were all less than 5\% (Fig. 2a and b), while at UVT $=75 \%$, the relative

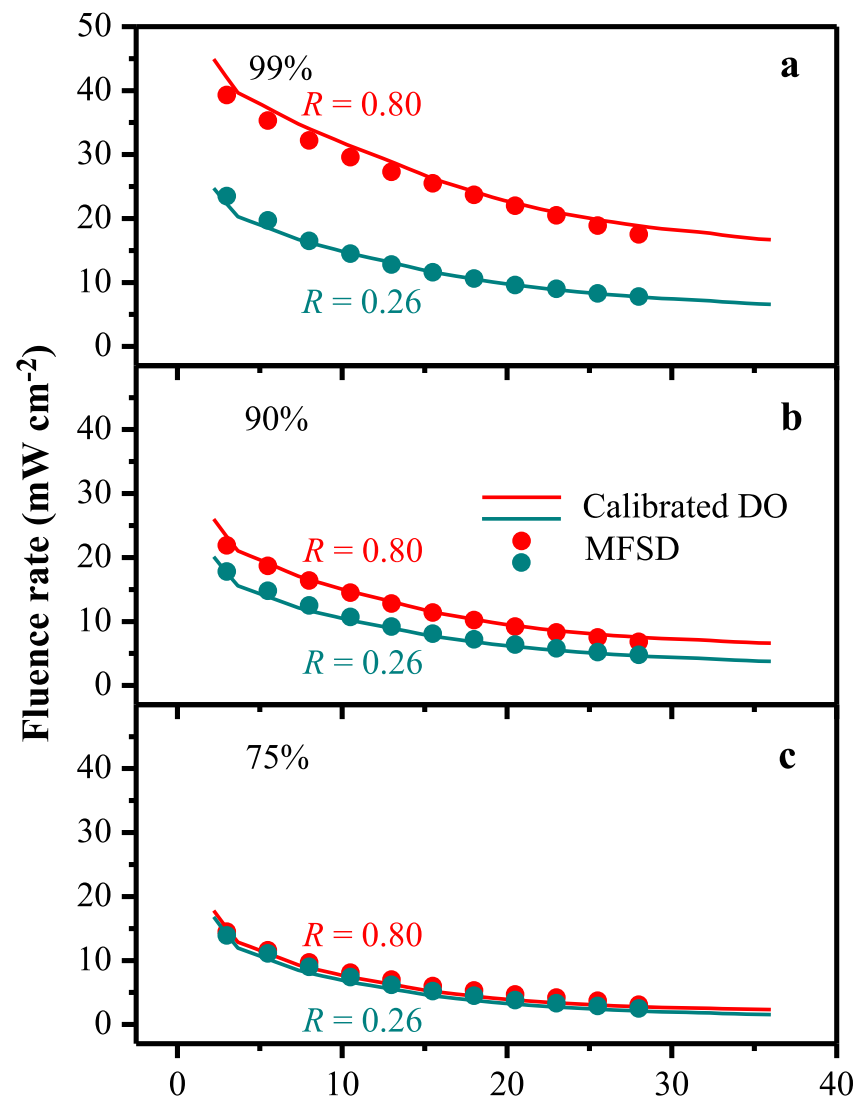

Radial distance from the sleeve ( $\mathrm{mm})$

Fig. 2. Radial fluence rate (FR) distributions in the central planes of UV reactors with two inner-wall reflectivities ( $R=0.26$ and 0.80$)$ at various water UVTs as determined by both the calibrated DO radiation model and the MFSD: (a) 99\%; (b) $90 \%$; and (c) $75 \%$. The diffuse fraction $\left(f_{\mathrm{d}}\right)$ was set at 0.1 in the calibrated DO radiation model. deviations became a little larger (about 13\%) (Fig. 2c), which is reasonable since the $\mathrm{CF}_{\mathrm{u}}$ was determined based on the $\mathrm{CF}_{\mathrm{i}}$ values at high UVTs. Good agreements between the predicted and the measured data were also found for the axial FR distributions, in both the near-lamp and the near-wall regions (data not shown). This demonstrates that the calibrated DO radiation model can be a reliable tool for UV radiation simulations in UV reactors with a reflective inner wall. The accurate prediction of FR distributions by the calibrated DO radiation model facilitated the following analysis of the effects of inner-wall reflection on FR distributions and REF.

\subsection{Effect of inner-wall reflection on FR distributions}

Fig. 3 shows the radial FR distributions in the central planes of UV reactors with two inner-wall reflectivities and three $f_{\mathrm{d}}$ values $(0.1,0.5$, and 0.9$)$ at various water UVTs. Compared to those with the ORW (i.e., $R=0.26$ ), the FRs in UV reactors with the HRW (i.e., $R=0.80$ ) increased significantly at high UVTs and even almost doubled at UVT $=99 \%$ (Fig. 3a and b). This is because at a higher UVT, more UV radiation reaches the inner wall and then is reflected back into the reactor chamber by the HRW. The effect of inner-wall diffuse reflection on FR distributions was negligible in UV reactors with the ORW (Fig. 3c and e); however, with the HRW at high UVTs,

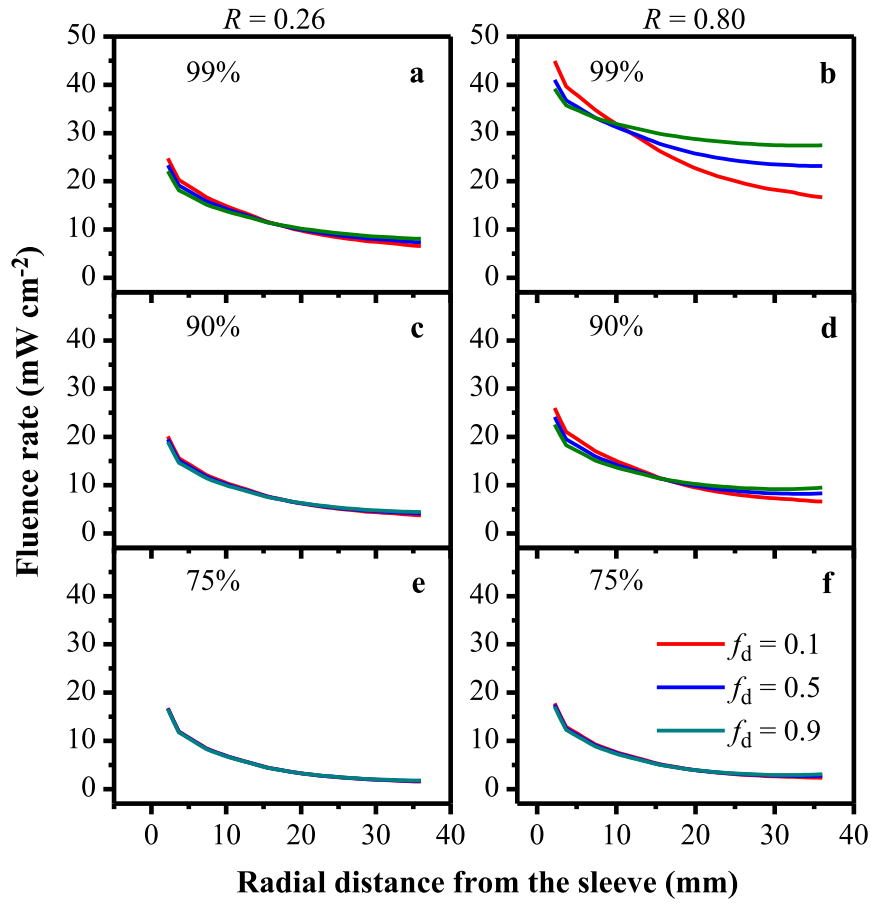

Fig. 3. Radial FR distributions in the central planes of UV reactors with two inner-wall reflectivities $(R=0.26$ and 0.80$)$ and three $f_{\mathrm{d}}$ values $(0.1,0.5$ and 0.9$)$ at various water UVTs: (a, b) $99 \%$; (c, d) $90 \%$; and (e, f) $75 \%$. 
the FR distributions became obviously more uniform as the $f_{\mathrm{d}}$ increased (Fig. 3b and d). This can be ascribed to the fact that the diffusely reflected UV light returned to the reactor chamber in all directions. Consequently, the optical path lengths were extended in the near-wall region but reduced in the near-lamp region, resulting in increased FRs near the wall and decreased FRs close to the lamp. These findings are in accordance with those reported by Li et al. (2012), and an improvement made here is that the effect of the diffuse reflection was demonstrated directly and quantitatively.

Table 2 shows the characteristic parameters of FR distributions in the central planes of UV reactors with two inner-wall reflectivities and three $f_{\mathrm{d}}$ values at various water UVTs. It was found that at $\mathrm{UVT}=99 \%$, both the area-averaged FR $\left(\mathrm{FR}_{\mathrm{aa}}\right)$ in the central planes and the volume-averaged FR $\left(\mathrm{FR}_{\mathrm{va}}\right)$ were more than doubled when the inner-wall reflectivity increased from 0.26 to 0.80 . For UV reactors with a higher inner-wall diffuse reflection (i.e., a greater $f_{\mathrm{d}}$ ), the relative standard deviation (RSD) of the cross-sectional FR decreased by having an increased minimum $F R\left(\mathrm{FR}_{\min }\right)$ and a decreased maximum FR (FR $\max )$. In the UV reactor with the HRW and $f_{\mathrm{d}}=0.9$ at $\mathrm{UVT}=99 \%$, the $\mathrm{FR}_{\min }$ even increased to be about $60 \%$ of the $\mathrm{FR}_{\max }$, leading to the lowest RSD of 0.138 . Therefore, diffuse reflection at high UVTs can greatly enhance the FR distribution uniformity in UV reactors with a highly reflective inner wall. A uniform FR distribution has been traditionally treated as a promoter for the UV reactor performance. It should be noted that the $\mathrm{FR}_{\mathrm{va}}$ also increased with increasing $f_{\mathrm{d}}$, with the largest increase being $19 \%$. This is because with a higher inner-wall diffuse reflection, more UV radiation was retained in the reactor chamber by having an increased axial optical path length. The elevated $F_{\mathrm{va}}$ can further improve the UV reactor performance.

\subsection{Effect of inner-wall reflection on REF}

The REFs of UV reactors with various inner-wall reflections are shown in Fig. 4. In either type of UV reactor (i.e., the L-shape or the U-shape), considerable REF increments were found with the HRW as compared to those with the ORW. For example, at UVT $=90 \%$, a typical UVT for drinking water treatment, the REF was raised by $42-64 \%$ for the L-shape UV reactors and by $44-55 \%$ for the U-shape UV reactors; and at UVT $=99 \%$, the REF was even raised by

Table 2

Characteristic parameters for FR distributions in the central planes and $\mathrm{FR}_{\mathrm{va}}$ values $\left(\mathrm{mW} \mathrm{cm}{ }^{-2}\right)$ of UV reactors with two inner-wall reflectivities and three $f_{\mathrm{d}}$ values $(0.1$, 0.5 and 0.9 ) at various water UVTs.

\begin{tabular}{|c|c|c|c|c|c|c|}
\hline \multirow[t]{2}{*}{ Parameter } & \multicolumn{3}{|c|}{$R=0.26$} & \multicolumn{3}{|c|}{$R=0.80$} \\
\hline & $f_{\mathrm{d}}=0.1$ & $f_{\mathrm{d}}=0.5$ & $f_{\mathrm{d}}=0.9$ & $f_{\mathrm{d}}=0.1$ & $f_{\mathrm{d}}=0.5$ & $f_{\mathrm{d}}=0.9$ \\
\hline \multicolumn{7}{|l|}{$\mathrm{UVT}=99 \%$} \\
\hline $\mathrm{FR}_{\mathrm{aa}}$ & 10.61 & 10.74 & 10.88 & 23.73 & 26.37 & 29.36 \\
\hline $\mathrm{FR}_{\max }$ & 32.55 & 30.91 & 29.44 & 52.88 & 47.82 & 45.44 \\
\hline $\mathrm{FR}_{\min }$ & 6.11 & 6.88 & 7.57 & 15.95 & 22.18 & 26.75 \\
\hline RSD & 0.566 & 0.496 & 0.434 & 0.390 & 0.220 & 0.138 \\
\hline $\mathrm{FR}_{\mathrm{va}}$ & 7.60 & 7.65 & 7.72 & 17.51 & 19.31 & 20.87 \\
\hline \multicolumn{7}{|l|}{$\mathrm{UVT}=90 \%$} \\
\hline $\mathrm{FR}_{\mathrm{aa}}$ & 7.08 & 7.11 & 7.14 & 10.58 & 10.92 & 11.24 \\
\hline $\mathrm{FR}_{\max }$ & 28.41 & 27.73 & 27.08 & 34.60 & 32.40 & 30.55 \\
\hline $\mathrm{FR}_{\min }$ & 3.44 & 3.79 & 4.11 & 6.13 & 7.76 & 8.89 \\
\hline RSD & 0.774 & 0.732 & 0.692 & 0.612 & 0.500 & 0.413 \\
\hline $\mathrm{FR}_{\mathrm{va}}$ & 5.05 & 5.06 & 5.08 & 7.51 & 7.72 & 7.91 \\
\hline \multicolumn{7}{|l|}{$\mathrm{UVT}=75 \%$} \\
\hline $\mathrm{FR}_{\mathrm{aa}}$ & 4.25 & 4.25 & 4.26 & 4.96 & 4.99 & 5.02 \\
\hline $\mathrm{FR}_{\max }$ & 25.98 & 25.84 & 25.73 & 27.06 & 26.62 & 26.21 \\
\hline $\mathrm{FR}_{\min }$ & 1.39 & 1.51 & 1.61 & 2.11 & 2.49 & 2.76 \\
\hline RSD & 1.227 & 1.212 & 1.198 & 1.070 & 1.026 & 0.986 \\
\hline $\mathrm{FR}_{\mathrm{va}}$ & 3.05 & 3.05 & 3.05 & 3.55 & 3.57 & 3.59 \\
\hline
\end{tabular}

$\mathrm{FR}_{\mathrm{aa}}$ : area-averaged FR $\left(\mathrm{mW} \mathrm{cm} \mathrm{cm}^{-2}\right) ; \mathrm{FR}_{\max }$ : maximum FR $\left(\mathrm{mW} \mathrm{cm}^{-2}\right) ; \mathrm{FR}_{\min }: \min -$ imum FR $\left(\mathrm{mW} \mathrm{cm}^{-2}\right)$; and RSD: relative standard deviation.

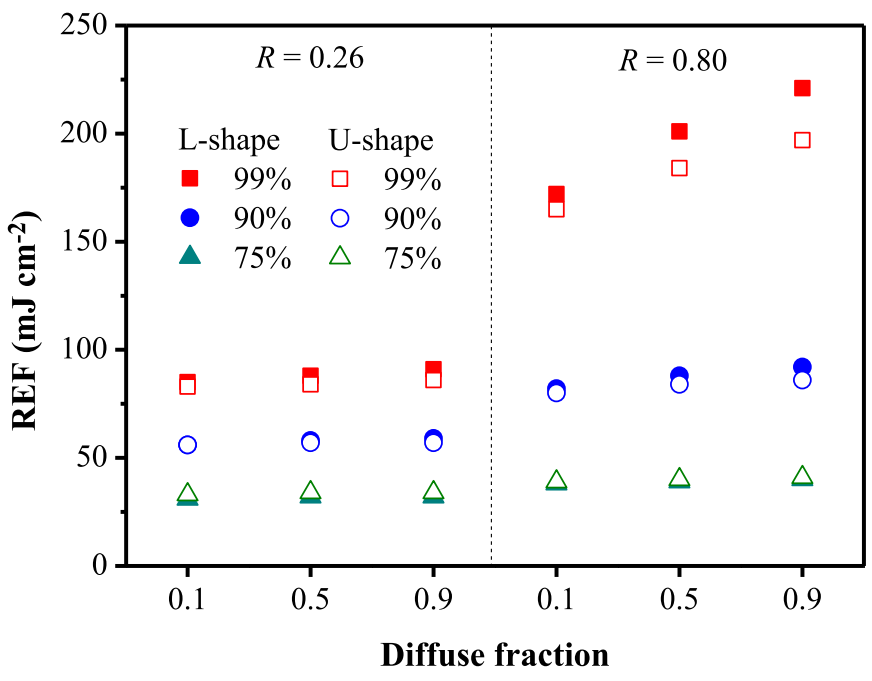

Fig. 4. REFs of UV reactors with two inner-wall reflectivities $(R=0.26$ and 0.80$)$ and three $f_{\mathrm{d}}$ values $(0.1,0.5$ and 0.9$)$ at various water UVTs. The solid and open symbols indicate the results of the L-shape and U-shape UV reactors, respectively.

103-161\% and 99-138\%, respectively. This can be attributed to the significantly elevated $\mathrm{FR}_{\mathrm{va}}$ values in UV reactors with the HRW. In fact, the REF increments in both types of UV reactors with the HRW were comparable to the $\mathrm{FR}_{\mathrm{va}}$ increments, which were $49-57 \%$ at UVT $=90 \%$ and $130-175 \%$ at UVT $=99 \%$ (Table 2 ). Chen et al. (2011) found a $24 \%$ increment in the microbial log inactivation at UVT $=92 \%$ when the inner-wall reflectivity increased from 0 to 0.6 in a cross-flow UV reactor. This increment is relatively lower as compared to the results in this study, mainly arising from a much larger inlet water velocity (i.e., $0.86 \mathrm{~m} \mathrm{~s}^{-1}$ ) adopted in their study, which resulted in a flush flow in the reactor chamber that could not make full use of the reflected UV radiation.

As the $f_{\mathrm{d}}$ increased, the REF increments in UV reactors with the ORW were negligible (i.e., $<7 \%$ ), while those in UV reactors with the HRW were as large as $19-28 \%$. The effect of $f_{\mathrm{d}}$ on the reactor performance can also be seen from the corresponding fluence distributions (Fig. S3). For UV reactors with the HRW, the fluence distributions obviously shifted to higher fluences as the $f_{\mathrm{d}}$ increased (Figs. S3a and b); by contrast, the fluence distributions of UV reactors with the ORW had little shift regardless of the $f_{\mathrm{d}}$ (Figs. S3c and $\mathrm{d}$ ). As mentioned above, both the enhanced FR distribution uniformity and the elevated $\mathrm{FR}_{\mathrm{va}}$ could contribute to the REF increment. To clarify their individual role, the ratios of the $\mathrm{FR}_{\mathrm{va}}$ and REF with $f_{\mathrm{d}}=0.5$ and 0.9 to those with $f_{\mathrm{d}}=0.1$ in UV reactors with the HRW at various water UVTs were analyzed (Fig. 5). It was found that at all the three UVTs, the percent increases of the REF in the Lshape UV reactors were a little higher than those of the $\mathrm{FR}_{\mathrm{va}}$; however, in the U-shape UV reactors they were almost the same. Taking the case of UVT $=99 \%$ for example, the $\mathrm{FR}_{\mathrm{va}}$ was increased by $21 \%$ in UV reactors with $f_{\mathrm{d}}=0.9$, while the percent REF increases were $28 \%$ and $19 \%$, respectively, in the L-shape and U-shape UV reactors. Therefore, the inner-wall diffuse reflection had varied effects on the UV reactor performance, which depended on the reactor configuration. For the U-shape UV reactors, the performance improvement was in line with the $\mathrm{FR}_{\mathrm{va}}$ increment resulting from the inner-wall diffuse reflection; while for the L-shape UV reactors, the enhanced FR distribution uniformity could have a further contribution to the UV reactor performance. This can be ascribed to the different hydrodynamics in the two types of UV reactors. As illustrated in Fig. S4, the flow pattern in the L-shape UV reactor was close to a plug flow. In this situation, the particle 


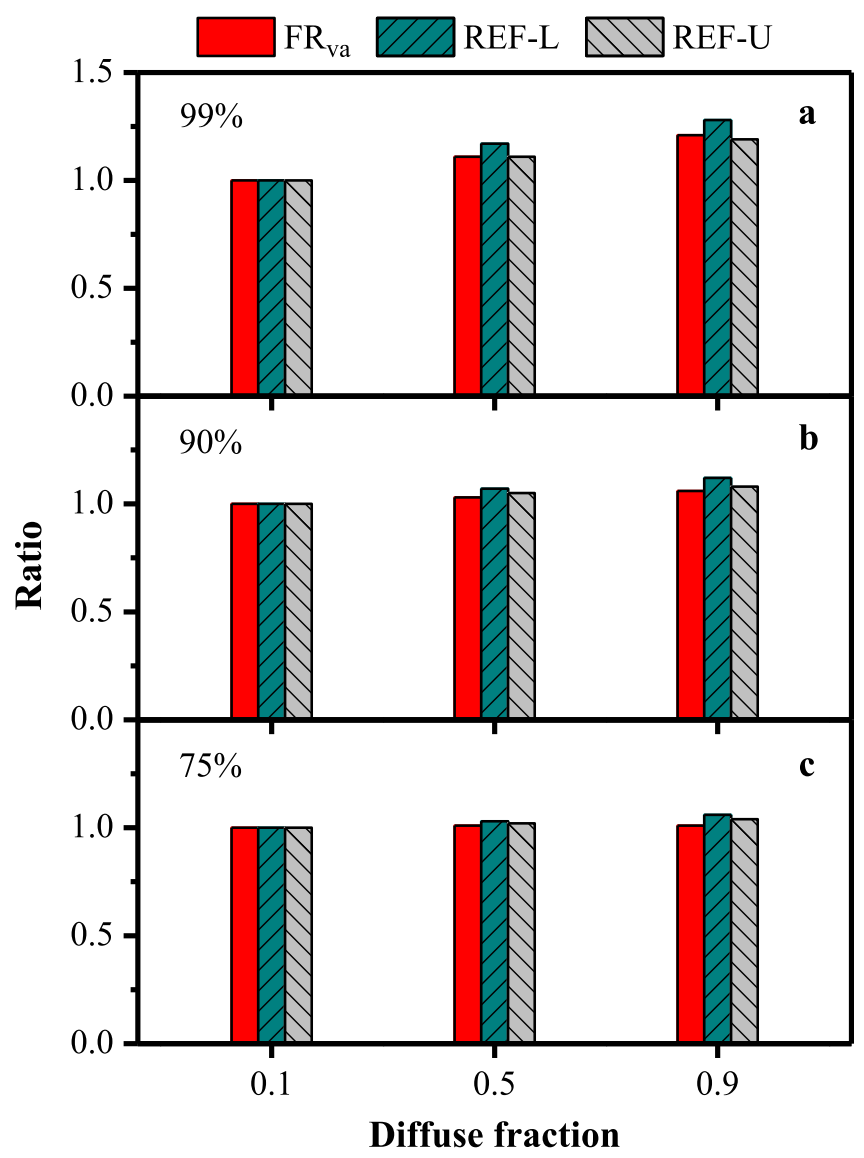

Fig. 5. Ratios of the volume-averaged FR ( $\left.\mathrm{FR}_{\mathrm{va}}\right)$ and $\mathrm{REF}$ with $f_{\mathrm{d}}=0.5$ and 0.9 to those with $f_{\mathrm{d}}=0.1$ in UV reactors with a highly reflective inner wall at various water UVTs: (a) 99\%; (b) 90\%; and (c) 75\%. The REF-L and REF-U represent the REFs of the L-shape and U-shape UV reactors, respectively.

minimum fluence $\left(F_{\min }\right)$, which plays a determinant role in the REF (Li et al., 2016), would most probably be found with particles traveling in the near-wall region. Because the $\mathrm{FR}_{\mathrm{va}}$ increment was a net result of the FR increase in the near-wall region subtracting the FR decrease in the near-lamp region, the degree of increase in the $F_{\min }$ was slightly greater than that in the $\mathrm{FR}_{\mathrm{va}}$. Consequently, the percent increase of the REF was a little higher than that of the $\mathrm{FR}_{\mathrm{va}}$. In contrast, the $F_{\min }$ in the U-shape UV reactor would most probably be found with particles in the flush flow that crossed over both the near-lamp and the near-wall regions. Consequently, the $F_{\min }$ would have a similar degree of increase to the $\mathrm{FR}_{\mathrm{va}}$, which resulted in similar percent increases of the REF and the $\mathrm{FR}_{\mathrm{va}}$.

\subsection{Contribution of FR distribution uniformity to UV reactor performance}

The result that the FR distribution uniformity had a limited contribution to the UV reactor performance is somewhat unexpected, since creating a uniform UV radiation has traditionally been viewed as an effective measure to improve the UV reactor performance (Schoenen, 1996). To confirm the above finding, a UV reactor with an utmost uniform FR distribution was created by making the inner wall $100 \%$ reflective (i.e., $R=1$ ) with a fully diffuse reflection (i.e., $f_{\mathrm{d}}=1$ ) (denoted as the FDR UV reactor), and its performance was compared to that of a common UV reactor with a partially diffuse reflection (i.e., $R=0.26, f_{\mathrm{d}}=0.1$ ) (denoted as the PDR UV reactor). The $\mathrm{FR}_{\mathrm{va}}$ in the two UV reactors was kept identical at
$6.30 \mathrm{~mW} \mathrm{~cm} \mathrm{~cm}^{-2}$ to rule out its interference. The radial FR distributions in the two UV reactors at UVT $=99 \%$ are shown in Fig. S5. Results indicate that the FRs in the central plane of the PDR UV reactor decreased dramatically from 32.55 to $6.11 \mathrm{~mW} \mathrm{~cm} \mathrm{~cm}^{-2}$ $(\mathrm{RSD}=0.576)$, while those of the FDR UV reactor only varied from 13.38 to $10.74 \mathrm{~mW} \mathrm{~cm}^{-2}$ (RSD $=0.050$ ).

The REFs of the PDR and FDR UV reactors at UVT $=99 \%$ with different flow rates are shown in Fig. 6. With the L-shape configuration, the FDR UV reactor had a $12 \%$ higher REF than the PDR UV reactor; while with the U-shape configuration, the two UV reactors had identical REFs (Fig. 6a). As the flow rate increased from 0.57 to $1.15 \mathrm{~m}^{3} \mathrm{~h}^{-1}$, the REF of the FDR UV reactor was increased by $19 \%$ as compared to that of the PDR UV reactor with the L-shape configuration; however, the REFs of the two UV reactors with the U-shape configuration were still the same (Fig. 6b). This is due to the fact that as the flow rate increased, the flow pattern became more like a plug flow in the L-shape UV reactor, while it remained as a mixed flow in the U-shape UV reactor. Therefore, the FR distribution uniformity made a conditioned contribution to the UV reactor performance. To be specific, in UV reactors with a plug-like flow (e.g., the L-shape UV reactor), the FR distribution uniformity contributed notably to the REF (12-19\%); while in UV reactors with a mixed flow (e.g., the U-shape UV reactor), the FR distribution uniformity had little influence on the REF. The different performances of the PDR and FDR UV reactors with L-shape and U-shape configurations were also manifested by their corresponding fluence distributions (Fig. S6). In accordance with the slightly higher REFs, the $F_{\min }$ values in the FDR UV reactors with the L-shape configuration were a little larger than those in the PDR UV reactors (Figs. S6a and c). On the other hand, no significant difference was found in the fluence distributions between the FDR and PDR UV

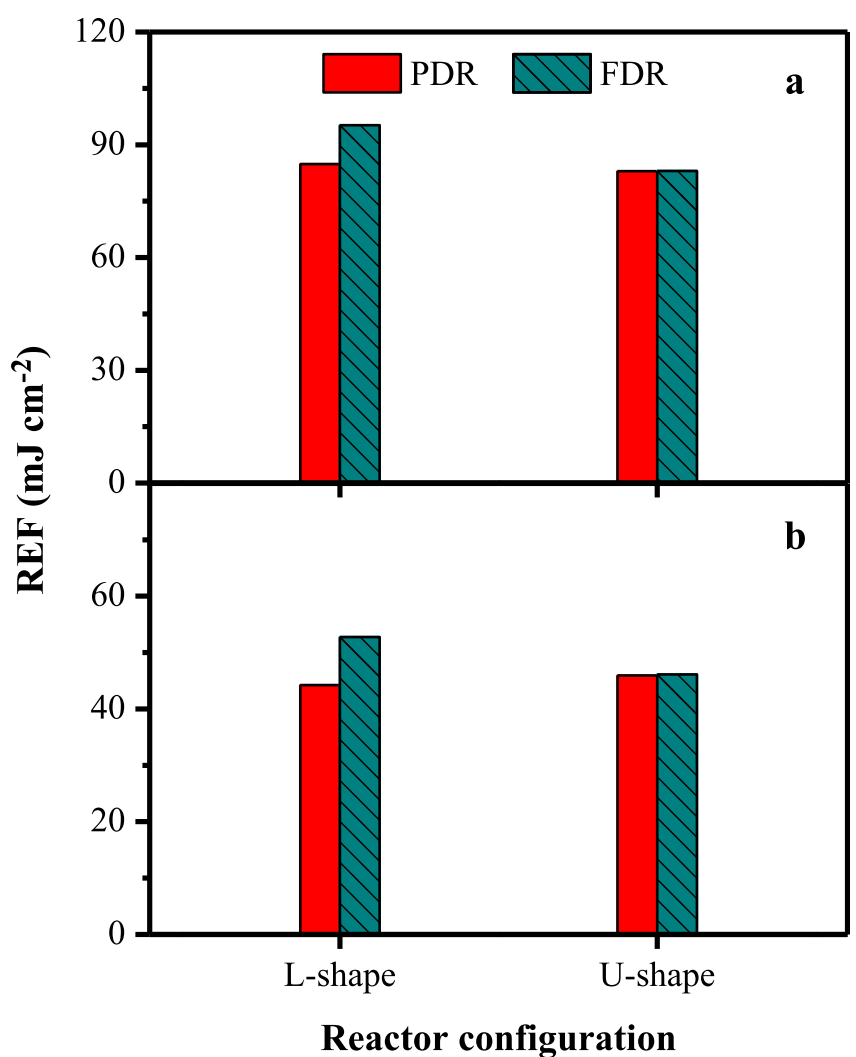

Fig. 6. REFs of PDR and FDR UV reactors with two configurations at water UVT $=99 \%$ at different flow rates: (a) $0.57 \mathrm{~m}^{3} \mathrm{~h}^{-1}$; and (b) $1.15 \mathrm{~m}^{3} \mathrm{~h}^{-1}$. 
reactors with the U-shape configuration (Figs. S6b and d), which agreed well with the REF results in Fig. 6 .

\subsection{Implications}

The inner-wall reflection in UV reactors can significantly increase the $\mathrm{FR}_{\mathrm{va}}$ and the corresponding REF, and the higher the inner-wall reflectivity is, the greater improvement of the UV reactor performance can be achieved. Therefore, employing a highly reflective inner wall can be an efficient approach to improve the UV reactor performance, which will eventually increase the UV reactor treatment capacity and reduce the specific cost. The reflective inner wall can be directly made of a highly reflective material, or be realized following the structure of UV reactors with UV-LEDs (i.e., a reflector is put behind a quartz tube).

The inner-wall diffuse reflection makes a further contribution to the UV reactor performance. Nevertheless, this benefit is only notable $(19-28 \%)$ with a highly reflective inner wall $(R=0.80)$ at $\mathrm{UVT}=99 \%$, and a higher $f_{\mathrm{d}}$ usually means an increased roughness, which causes the inner wall more likely to be fouled. As a result, it is not advisable to improve the UV reactor performance by increasing the inner-wall diffuse reflectivity. For the first time, the FR distribution uniformity has been proven to make a conditioned contribution to the UV reactor performance, and in common UV reactors with a mixed flow, the FR distribution uniformity has little impact on the UV reactor performance. Therefore, concerning the UV reactor design, more attention should be paid to the hydrodynamics and the $\mathrm{FR}_{\mathrm{va}}$ rather than the FR distribution uniformity.

\section{Conclusions}

The impact of inner-wall reflection on the UV reactor performance was evaluated in annular single-lamp UV reactors by using CFD simulations, with a special focus on the role of diffuse reflection. The FR distributions inside the UV reactors were simulated with a calibrated DO radiation model. The effects of inner-wall reflection on the FR distributions and REF were investigated, and the contribution of FR distribution uniformity to the UV reactor performance was clarified. The main conclusions included:

- The FR distributions in UV reactors with a reflective inner wall could be predicted accurately with the calibrated DO radiation model, with the relative deviations between the predicted and measured $\mathrm{FR}_{\mathrm{wa}}$ values being less than $5 \%$ at high water UVTs $(\geq 90 \%)$.

- The FRs in UV reactors with the HRW (i.e., $R=0.80$ ) were significantly higher than those with the ORW (i.e., $R=0.26$ ), especially at high water UVTs. The inner-wall diffuse reflection enhanced the FR distribution uniformity and increased the $\mathrm{FR}_{\mathrm{va}}$ at the same time.

- The REF increment of UV reactors with the HRW was considerable and highly related with the $\mathrm{FR}_{\mathrm{va}}$ increment. The innerwall diffuse reflection further increased the reactor REF, which mainly arose from the elevated $\mathrm{FR}_{\mathrm{va}}$.

- The FR distribution uniformity had conditioned contributions to the UV reactor performance. In the L-shape UV reactors with a plug-like flow, the FR distribution uniformity contributed notably to the REF increment (12-19\%); however, in the Ushape UV reactors with a mixed flow, the FR distribution uniformity had little influence on the REF.

\section{Acknowledgments}

The authors gratefully acknowledge the financial support from the National Natural Science Foundation (51290281, 51525806, 51408592) and the Ministry of Science and Technology (2012ZX07404-004) of China.

\section{Appendix A. Supplementary data}

Supplementary data related to this article can be found at http:// dx.doi.org/10.1016/j.watres.2016.11.068.

\section{References}

ANSYS, 2010. ANSYS FLUENT 13.0 Theory Guide. ANSYS Inc., Canonsburg, PA, USA Chen, J., Deng, B.Q., Kim, C.N., 2011. Computational fluid dynamics (CFD) modeling of UV disinfection in a closed-conduit reactor. Chem. Eng. Sci. 66 (21), 4983-4990.

Chiu, K., Lyn, D.A., Savoye, P., Blatchley III, E.R., 1999. Effect of UV system modifications on disinfection performance. J. Environ. Eng. 125 (5), 459-469.

Esplugas, S., Gimenez, J., Contreras, S., Pascual, E., Rodriguez, M., 2002. Comparison of different advanced oxidation processes for phenol degradation. Water Res. 36 (4), 1034-1042.

Hessling, M., Gross, A., Hoenes, K., Rath, M., Stangl, F., Tritschler, H., Sift, M., 2016. Efficient disinfection of tap and surface water with single high power $285 \mathrm{~nm}$ LED and square quartz tube. Photonics 3 (1), 7.

Hijnen, W.A.M., Beerendonk, E.F., Medema, G.J., 2006. Inactivation credit of UV radiation for viruses, bacteria and protozoan (oo) cysts in water: a review. Water Res. 40 (1), 3-22.

Ho, C.K., 2009. Evaluation of reflection and refraction in simulations of ultraviolet disinfection using the discrete ordinates radiation model. J. Water Sci. Technol. 15 (12), 2421-2428.

Ho, C.K., Khalsa, S.S., Wright, H.B., Wicklein, E., 2009. Modeling UV disinfection using integrated computational fluid dynamics and discrete ordinates radiation models. Proc. Water Environ. Fed. 2009 (1), 257-273.

Li, M.K., Qiang, Z.M., Li, T.G., Bolton, J.R., Liu, C.L., 2011. In situ measurement of UV fluence rate distribution by use of a micro fluorescent silica detector. Environ. Sci. Technol. 45 (7), 3034-3039.

Li, M.K., Qiang, Z.M., Bolton, J.R., Ben, W.W., 2012. Impact of reflection on the fluence rate distribution in a UV reactor with various inner walls as measured using a micro-fluorescent silica detector. Water Res, 46 (11), 3595-3602.

Li, W.T., Li, M.K., Bolton, J.R., Qiang, Z.M., 2016. Configuration optimization of UV reactors for water disinfection with computational fluid dynamics: feasibility of using particle minimum UV dose as a performance indicator. Chem. Eng. J. 306, $1-8$.

Liu, D., Ducoste, J.J., Jin, S., Linden, K., 2004. Evaluation of alternative fluence rate distribution models. Aqua J. Water Supply Res. Technol. 53 (6), 391-408.

Qiang, Z.M., Li, M.K., Bolton, J.R., Qu, J.H., Wang, C., 2013. Estimating the fluence delivery in UV disinfection reactors using a 'detector-model' combination method. Chem. Eng. J. 233, 39-46.

Schoenen, D., 1996. The influence of inhomogenous irradiation in UV disinfectionexperimental findings and theoretical considerations. J. Water Supply Res. Technol. AQUA 45 (3), 20-129.

Sommer, R., Cabaj, A., Haider, T., 1996. Microbicidal effect of reflected UV radiation in devices for water disinfection. Water Sci. Technol. 34 (7-8), 173-177.

Sozzi, D.A., Taghipour, F., 2006a. UV reactor performance modeling by Eulerian and Lagrangian methods. Environ. Sci. Technol. 40 (5), 1609-1615.

Sozzi, D.A., Taghipour, F., 2006b. Computational and experimental study of annular photo-reactor hydrodynamics. Int. J. Heat. Fluid Fl 27 (6), 1043-1053.

Sozzi, A., Taghipour, F., 2007. The importance of hydrodynamics in UV advanced oxidation reactors. Water Sci. Technol. 55 (12), 53-58.

Wols, B.A., Hofman, J.A.M.H., Beerendonk, E.F., Uijttewaal, W.S.J., van Dijk, J.C., 2011 A systematic approach for the design of UV reactors using computational fluid dynamics. AIChE J. 57 (1), 193-207.

Wurtele, M.A. Kolbe, T, Lipsz, M., Kulberg A., Weyers, M., Kneissl, M., Jekel, M. 2011. Application of GaN-based ultraviolet-C light emitting diodes-UV LEDs-for water disinfection. Water Res. 45 (3), 1481-1489.

Xu, B., Gao, N.Y., Cheng, H.F., Xia, S.J., Rui, M., Zhao, D.D., 2009. Oxidative degradation of dimethyl phthalate (DMP) by UV/ $\mathrm{H}_{2} \mathrm{O}_{2}$ process. J. Hazard. Mater. 162 (2-3), 954-959.

Xu, C., Zhao, X.S., Rangaiah, G.P., 2013. Performance analysis of ultraviolet water disinfection reactors using computational fluid dynamics simulation. Chem. Eng. J. 221, 398-406. 